\title{
Total Removal of an Intramedullary Meningioma of the Medulla Oblongata \\ -Case Report-
}

\author{
Saburo SAKAKI, Tamotsu OHSUGI, Takahiro CHAKI, \\ Masahiro SHIRAISHI, Kenzo MATSUOKA and Soei GO* \\ Departments of Neurological Surgery and \\ *Pathology, Ehime University School of Medicine, Ehime
}

\begin{abstract}
A 12-year-old boy had central von Recklinghausen's neurofibromatosis with multiple intracranial meningiomas and bilateral acoustic neurinomas. One of the meningiomas was found in the parenchyma of the medulla oblongata and was successfully removed. To our knowledge, this is the first reported case of surgical removal of an intramedullary meningioma of the medulla oblongata. Details of the clinical, neuroradiological, and operative findings are described.
\end{abstract}

Key words: intramedullary meningioma, medulla oblongata, neurofibromatosis, childhood, brain neoplasms

\section{Introduction}

Although intracranial meningiomas in children are rare, ${ }^{6,15,26)}$ meningiomas are relatively common among children with central von Recklinghausen's neurofibromatosis. ${ }^{7,21)}$ Recently, we encountered the extremely rare instance of neurofibromatosis with multiple meningiomas and bilateral acoustic neurinomas. One of the meningiomas was entirely embedded in the parenchyma of the medulla oblongata and was successfully removed. We believe this to be the first reported case of surgical removal of an intramedullary meningioma of the medulla oblongata.

\section{Case Report}

This 12-year-old male patient was the younger of uniovular twins. The older twin had multiple meningiomas $-\mathbf{a}$ frontotemporal convexity meningioma, a foramen magnum meningioma, and a choroid plexus meningioma in the right lateral ventricle - and bilateral acoustic neurinomas. Also, the older twin was blind in the right eye because of congenital maldevelopment of the papilla. There were no other known abnormalities in his family. The patient was admitted to Ehime University Hospital on February 22, 1985 for evaluation of intracranial lesions, although his only complaint was blindness in the right eye.

He was fully active and had no neurological abnormalities. He was blind in the right eye as a result of congenital maldevelopment of the papilla. There were several unpigmented cutaneous nodules in the right hypochondrial region, and biopsies of these nodules yielded a histological diagnosis of neurofibromatosis. Routine blood studies and chromosomal analysis were normal. Computed tomography (CT) showed moderately high density lesions with homogeneous enhancement, one in the right frontal parasagittal region $(4.8 \times 4.5 \mathrm{~cm})$ and another in the region of the foramen magnum $(1.7 \times 1.3 \mathrm{~cm})$ (Fig. 1). The location of the latter was accurately demonstrated on coronal and sagittal reconstruction

Received June 11, 1986; Accepted April 6, 1987

Authors' present addresses: T. Ohsugi, M.D., Department of Neurological Surgery, Uwajima City Hospital, Uwajima, Ehime, Japan; T. Chaki, M.D., Department of Neurological Surgery, Imabari Municipal Hospital, Imabari, Ehime, Japan. 

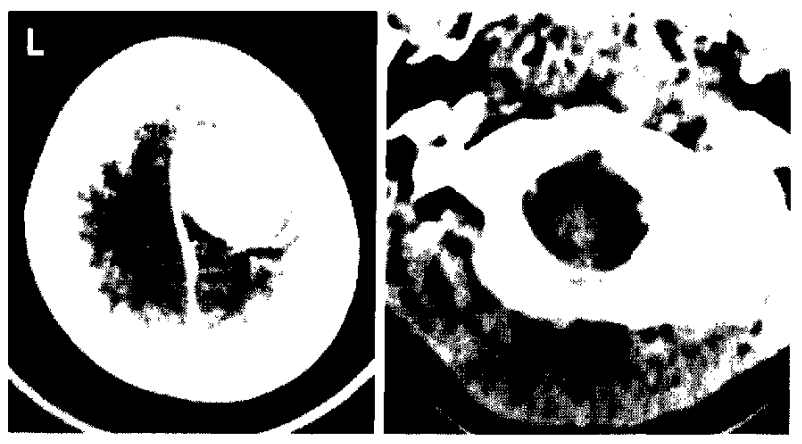

Fig. 1 CT scan showing moderately high density lesions with homogeneous enhancement. left: A $4.8 \times 4.5 \mathrm{~cm}$ mass in the right frontal parasagittal region. right: A $1.7 \times 1.3 \mathrm{~cm}$ mass in the region of the foramen magnum.
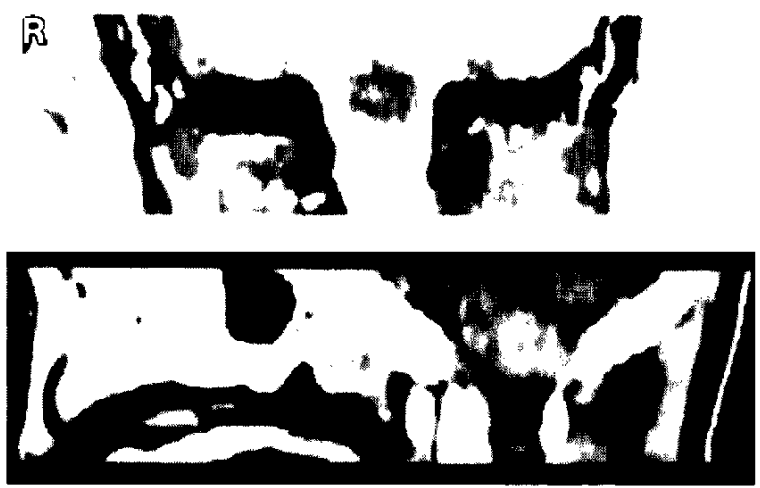

Fig. 2 Coronal (upper) and sagittal (lower) reconstruction views accurately showing the location of the tumor in the region of the foramen magnum and indicating an intramedullary tumor of the medulla oblongata.
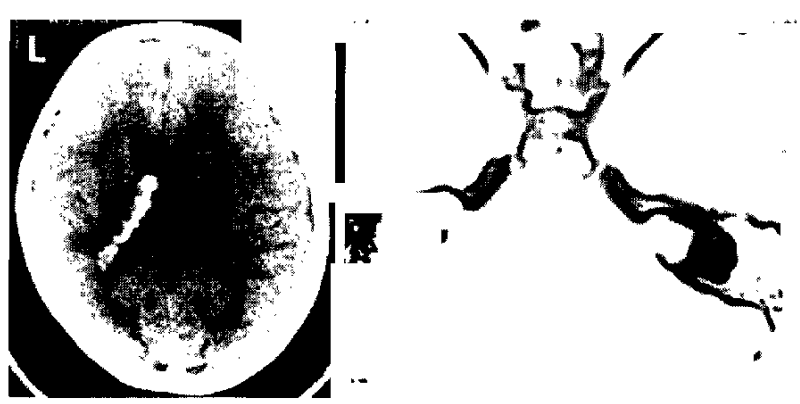

Fig. 3 left: A calcified lesion is seen in the left lateral ventricle, suggesting an intraventricular meningioma. right: The bilateral internal acoustic meatus are markedly dilated, suggesting bilateral acoustic neurinomas.
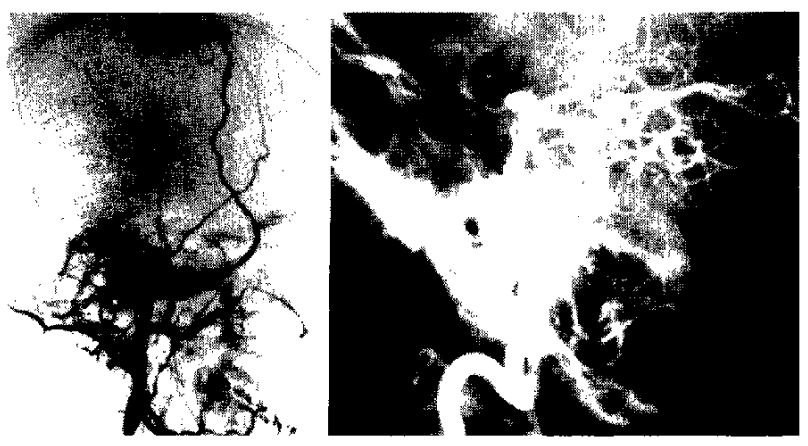

Fig. 4 left: Right carotid angiogram shows tumor staining of the right middle meningeal artery in the right parasagittal region. right: Vascularity in the region of the foramen magnum is not abnormal, on vertebral angiogram.

views, which indicated that it was an intramedullary tumor of the medulla oblongata (Fig. 2). A calcified lesion was also found in the left lateral ventricle. In addition to these multiple meningiomas, there were slightly dense, enhanced lesions in the bilateral, dilated internal acoustic meatus, suggestive of acoustic neurinomas (Fig. 3). Angiograms showed tumor staining of the right middle meningeal artery in the right frontal region, but no abnormal vascularity in the region of the foramen magnum (Fig. 4).

The preoperative diagnosis was multiple meningiomas (right parasagittal meningioma and choroid plexus meningioma of the left lateral ventricle), intramedullary tumor of the medulla oblongata, and bilateral acoustic neurinomas associated with von Recklinghausen's neurofibromatosis.

First operation: On March 18, 1985, a suboccipital craniectomy with a laminectomy of the $\mathrm{Cl}$ was performed through a midline skin incision with the patient in a prone position. The exposed dura mater over the cerebellum and the upper cervical spinal cord appeared normal and had good pulsation. The dura mater was opened with a Y-shaped incision. The medulla oblongata was markedly swollen, with a normal surface, which suggested an intramedullary tumor (Fig. 5). We separated and retracted the cerebellar tonsils and found the caudal portion of the floor of the fourth ventricle close to the obex to be bulging, with dark red discoloration. However, the surface of the ventricular floor appeared to be completely covered by an ependymal layer. The choroid plexus of the fourth ventricle did not adhere to the bulging floor. The medulla oblongata was incised to about $1.3 \mathrm{~cm}$ caudally from the obex on the midline, where we discovered a well demarcated, finely nodular tumor less than $2 \mathrm{~mm}$ from the surface of 

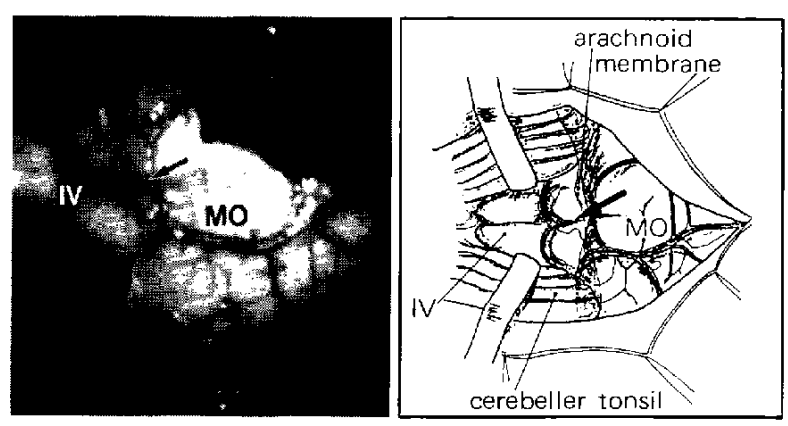

Fig. 5 left: Intraoperative photograph showing the swollen medulla oblongata (MO) with a normal surface. The floor of the fourth ventricle (IV) looks normal in the left side of the picture. The arrow indicates the obex. right: Schematic drawing of the operative findings.

the medulla oblongata.

Having received the frozen section diagnosis of meningioma during the operation, we decided to totally remove the tumor, which was solid and encapsulated. Soft, gliotic tissue with numerous fine vessels separated the tumor capsule from the surrounding neural tissue, providing a plane for dissection of the tumor. While carefully monitoring cardiac function and blood pressure, we removed the tumor completely, piece by piece. The pathological diagnosis was meningotheliomatous meningioma (Fig. 6).

The patient awoke from anesthesia immediately after the operation. His respiration was somewhat shallow but regular and spontaneous, so the endotracheal tube was left in place for several hours postoperatively. By the following day, his respiration had recovered and his vital and neurological functions were all normal.

Second operation: We performed a right frontotemporal craniotomy on May 2, 1985 and removed the entire parasagittal meningioma en bloc. The pathological diagnosis was meningotheliomatous meningioma.

The patient had mild hemiparesis following the second surgery, but it gradually improved and he was discharged on June 2, 1985 with no neurological deficits.

\section{Discussion}

Intracranial meningiomas in children are rare. Ingraham and Matson ${ }^{15)}$ found only three meningiomas $(0.4 \%)$ among 750 intracranial tumors in children under 14 years of age. Taptas, ${ }^{26)}$ in a review,

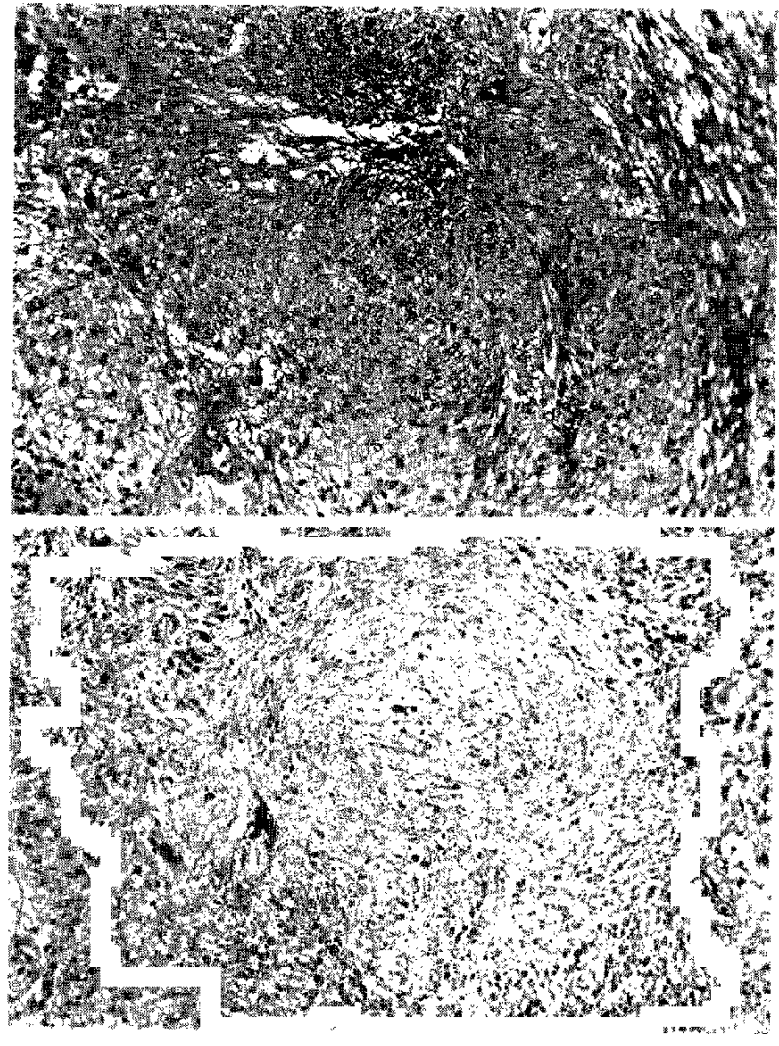

Fig. 6 Photomicrographs of the intramedullary tumor of the medulla oblongata. The tumor consists of poorly defined oval or spindle-shaped cells. The nuclei are relatively large and spheroidal. The tumor cells are arranged in sheets and are divided by a small amount of connective tissue (upper). There are interlacing bundles of long spindle-shaped cells (lower). HE stains, $\times 200$.

reported that there were 19 meningiomas (1.1\%) among 1760 brain tumors in children. Childhood meningiomas often arise in association with von Recklinghausen's neurofibromatosis. Crouse and Berg ${ }^{7}$ reported three meningiomas associated with von Recklinghausen's neurofibromatosis among 13 children and adolescents with primary meningeal tumors. Merten et al..$^{21)}$ discovered, in data collected from medical centers in the United States and Canada, 11 cases of neurofibromatosis among 48 children and adolescents with meningiomas. Of particular interest is that five of the six cases of multiple meningiomas in their series were associated with neurofibromatosis. Rodriguez and Berthrong ${ }^{24)}$ reviewed 49 cases of von Recklinghausen's neurofibromatosis with single or bilateral acoustic neurinomas and multiple meningiomas and found that six of 45 patients $(13.3 \%)$ were in the second decade, the youngest being 16 years old. Forty of $49 \mathrm{pa}$ - 
tients $(81.6 \%)$ had bilateral acoustic neurinomas, 26 of $48(54.2 \%)$ had intracranial meningiomas, 20 $(41.7 \%)$ had intracranial and intraspinal meningiomas, and eight of the last group had intraventricular meningiomas in addition to the intracranial and intraspinal meningiomas. ${ }^{81}$

Our patient had von Recklinghausen's neurofibromatosis and multiple meningiomas: right frontal parasagittal and left intraventricular meningiomas, and an intramedullary meningioma of the medulla oblongata associated with bilateral acoustic neurinomas. The intraventricular meningioma and neurinomas were not, however, histologically verified. To our knowledge, there has been no previous report of surgical excision of an intramedullary meningioma of the medulla oblongata in patients with or without von Recklinghausen's neurofibromatosis.

According to Lichtenstein, ${ }^{18}$ the basic pathological lesions of von Recklinghausen's neurofibromatosis are foci of hyperplasia and neoplasia of the supportive derivatives of the primitive ectoderm in association with hyperplasia of mesodermal elements. ${ }^{4,10,18,24,27)}$ When meningiomas occur, they are commonly multiple and adherent to the inner aspect of the dura mater. They frequently occur in the choroid plexus. Focal areas of hyperplasia or neoplasia are also observed in the cerebrospinal arachnoid. In our patient, it was difficult to identify the origin of the tumor, since it had already grown quite large. It was entirely contained in the lower medulla oblongata, the dorsal surface of which was markedly swollen and thinly covered with brain parenchyma. The bulging caudal floor of the fourth ventricle had a normal ependymal layer. During its dissection the tumor adhered strongly to the surrounding neural tissue. Its feeding and draining vessels were just under the obex, which is perhaps where tumor originated. It likely arose from the inferior tela choroidea or from the meningeal structures around the obex and developed in the parenchyma of the medulla oblongata. ${ }^{1,5.25,28)}$

Surgical intervention in patients with brain stem tumors is controversial. However, since the introduction of steroid therapy and the operating microscope, some neurosurgeons have biopsied such tumors and used the histological diagnosis to decide the appropriate irradiation therapy. ${ }^{2,9.14,23)}$ Also, many patients have been successfully treated for large neoplastic cysts in the brain stem by surgical evacuation followed by radiation therapy. ${ }^{11,16,17,19,22)}$ In our clinic, the primary treatment for brain stem tumors has been radiation therapy, except when the neuroradiological findings indicate benign, well demarcated tumors or cystic lesions. In the patient reported here we totally removed an intramedullary meningioma from the medulla oblongata without causing neurological deficits. The tumor mainly involved the lower and probably the dorsal portions of the medulla oblongata. There was a soft, gliotic interface between the tumor capsule and the functionally important brain parenchyma; thus, a dissecting plane was easily found with the aid of a surgical microscope and the small vascular adhesions were safely cut with microsurgical instruments. ${ }^{12,13,20)}$ For these reasons we did not encounter abnormal changes in vital functions during or after surgery. ${ }^{3)}$

We conclude that this surgical approach can be safely attempted in patients with brain stem tumors, provided the tumor is well demarcated and mainly involves the lower medulla oblongata, preferably dorsal to the central canal.

\section{References}

1) Abraham $\mathbf{J}$, Chandy $\mathbf{J}$ : Meningioma of the posterior fossa without dural attachment. A case report. $J$ Neurosurg 20: 177-179, 1963

2) Albright AL, Price RA, Guthkelch AN: Brain stem gliomas of children. A clinicopathological study. Cancer 52: 2313-2319, 1983

3) Baker GS: Physiologic abnormalities encountered after removal of brain tumors from the floor of the fourth ventricle. $J$ Neurosurg 23: 338-343, 1965

4) Canale D, Bebin J, Knighton RS: Neurologic manifestations of von Recklinghausen's disease of the nervous system. Confin Neurol 24: 359-403, 1964

5) Chaffee B, Donaghy RMP: Meningioma of the fourth ventricle. I Neurosurg 20: 520-522, 1963

6) Chan RC, Thompson GB: Intracranial meningiomas in childhood. Surg Neurol 21: 319-322, 1984

7) Crouse SK, Berg BO: Intracranial meningiomas in childhood and adolescence. Neurology (Minneap) 22: 135-141, 1972

8) Davidoff LM, Martin J: Hereditary combined neurinomas and meningiomas. I Neurosurg 12: 375384,1955

9) DeSousa AL, Kalsbeck JE, Mealey J J, Campbell RL, Hockey A: Intraspinal tumors in children. A review of 81 cases. J Neurosurg 51: 437-445, 1979

10) Fienman NL, Yakovac WC: Neurofibromatosis in childhood, J Pediatr 76: 339-346, 1970

11) Garcia CA, McGarry PA, Collada M: Ganglioglioma of the brain stem. Case report. J Neurosurg 60: 431434,1984

12) Garrido E, Stein BM: Microsurgical removal of intramedullary spinal cord tumors. Surg Neurol 7: 215219, 1977

13) Hakuba A, Nishimura S, Mishima Y, Kawano K: Foramen magnum tumors. Report of 21 cases. Neurol Med Chir (Tokyo) 22: 563-576, 1982

14) Hoffman HJ, Becker L, Craven MA: A clinically and 
pathologically distinct group of benign brain stem gliomas. Neurosurgery 7: 243-248, 1980

15) Ingraham FD, Matson DD: Neurosurgery of Infancy and Childhood. Springfield, CC Thomas, 1969, pp 624-631

16) Lassiter KRL, Alexander E Jr, Davis $\mathrm{CH} \mathrm{Jr}$, Kelly DL Jr: Surgical treatment of brain stem gliomas. $J$ Neurosurg 34: 719-725, 1971

17) Leal $O$, Miles J: Epidermoid cyst in the brain stem. Case report. $J$ Neurosurg 48: 811-813, 1978

18) Lichtenstein BW: Neurofibromatosis (von Recklinghausen's disease of the nervous system). Analysis of the total pathologic picture. Arch Neurol Phychiat 62: $822-839,1949$

19) Littman P, Jarrett P, Bilaniuk LT, Rorke LB, Zimmerman RA, Bruce DA, Carabell SC, Schut L: Pediatric brain stem gliomas. Cancer 45: 2787-2792, 1980

20) Malis LI: Intramedullary spinal cord tumors. Clin Neurosurg 25: 512-539, 1978

21) Merten DF, Gooding CA, Newton TH, Malamud N: Meningiomas of childhood and adolescence. $J$ Pediatr 84: 696-700, 1974

22) Pool JL: Gliomas in the region of the brain stem. $J$ Neurosurg 29: 164-167, 1968

23) Reigel DH, Scarff TB, Woodford JE: Biopsy of pediatric brain stem tumors. Childs Brain 5: 329-340, 1979

24) Rodriguez HA, Berthrong $M$ : Multiple primary intracranial tumors in von Recklinghausen's neurofibromatosis. Arch Neurol (Chicago) 14: 467-475, 1966

25) Schaerer JP, Woolsey RD: Intraventricular meningiomas of the fourth ventricle. I Neurosurg 17: 337341,1960

26) Taptas JN: Intracranial meningioma in a fourmonth-old infant simulating subdural hematoma. $J$ Neurosurg 18: 120-121, 1961

27) Terao H, Tsukamoto $Y$, Sano K: Intracranial tumors associated with von Recklinghausen's disease. No To Shinkei 19: 1235-1245, 1967 (in Japanese)

28) Vogel FS, Stevenson LD: Meningothelial meningioma of the fourth ventricle. $J$ Neuropath Exp Neurol 9: 443-448, 1950

Address reprint requests to: S. Sakaki, M.D., Department of Neurological Surgery, Ehime University School of Medicine, Shizukawa, Shigenobu-cho, Onsen-gun, Ehime 791-02, Japan. 